\title{
Unilateral hamstring foam rolling does not impair strength but the rate of force development of the contralateral muscle
}

\author{
Xin Ye ${ }^{\text {Corresp., } 1}{ }^{1}$ Benjamin S Killen ${ }^{2}$, Krista L Zelizney $^{3}$, William M Miller ${ }^{1}$, Sunggun Jeon ${ }^{1}$ \\ ${ }^{1}$ Department of Health, Exercise Science, and Recreation Management, University of Mississippi, University, Mississippi, United States \\ 2 Department of Health Related Professions, University of Mississippi Medical Center, Jackson, Mississippi, United States \\ 3 Nicole Wertheim College of Nursing \& Health Sciences, Florida International University, Miami, Florida, United States \\ Corresponding Author: Xin Ye \\ Email address: xye1@olemiss.edu
}

Background. Self-administered foam rolling (SAFR) is an effective massage technique often used in sport and rehabilitation settings to improve range of motion (ROM) without impairing the strength performance. However, the effects of unilateral SAFR on contralateral non-intervened muscle's rate of force development (RFD) are unknown. Therefore, the purpose of this investigation was to examine the acute effects of unilateral hamstrings SAFR on the contralateral limb flexibility, the isometric strength, and the RFD parameters. Methods. Thirty-four subjects (21 women) completed two separate randomly sequenced experimental visits, during which the control (rested for 10 minutes) or ten, 30second SAFR were performed with the dominant hamstring muscle group. Before (Pre) and after (Post) the interventions, the contralateral hip flexion passive ROM, the maximal explosive isometric strength of the contralateral knee flexors with the corresponding prime mover muscles' surface electromyographic (EMG) amplitude were measured. Separate two-way (time $x$ intervention) repeated measures analyses of variance (ANOVAs) were used to examine the potential changes of the dependent variables. Results. The SAFR significantly improved the contralateral limb ROM (Pre vs. Post: $68.3 \pm 21.0$ vs. $73.2 \pm$ 23.2 degrees, $p<0.001 ; d=0.22$ ). No change was found for the contralateral isometric strength or the maximal EMG amplitude. For the RFD parameters, the percent changes of the RFDs for the first 50, 100, and $200 \mathrm{~ms}$ of the maximal explosive isometric contraction were $-31.2 \%,-16.8 \%$, and $-10.1 \%$, respectively, following the unilateral SAFR, relative to the control condition. In addition, the decrement of the first 50-ms RFD reached statistical significance ( $p=0.007$; Cohen's $d=0.44$ ). Conclusion: Ten sets of 30-second unilateral hamstring SAFR improved the ROM of the non-intervened contralateral limb, but decreased its ability to generate force, especially during the early phase (e.g., $50 \mathrm{~ms}$ ) of the maximal explosive contraction. 


\section{Unilateral hamstring foam rolling does not impair}

2 strength but the rate of force development of the

3 contralateral muscle

Xin Ye ${ }^{1}$, Benjamin S. Killen ${ }^{2}$, Krista L. Zelizney ${ }^{3}$, William M. Miller ${ }^{1}$, Sunggun Jeon ${ }^{1}$

${ }^{1}$ Department of Health, Exercise Science, and Recreation Management, University of

8 Mississippi, University, Mississippi, USA

$9{ }^{2}$ Department of Health Related Professions, University of Mississippi Medical Center, Jackson,

10 Mississippi, USA

$11{ }^{3}$ Nicole Wertheim College of Nursing \& Health Sciences, Florida International University,

12 Miami, Florida, USA

Corresponding Author:

15 Xin Ye, Ph.D., CSCS*D, ACSM-CEP

16 Assistant Professor

17 Department of Health, Exercise Science, and Recreation Management

18 The University of Mississippi

19236 Turner Center

20 University, Mississippi, USA

21 Email address: xye1@olemiss.edu 


\section{Abstract}

Background. Self-administered foam rolling (SAFR) is an effective massage technique often used in sport and rehabilitation settings to improve range of motion (ROM) without impairing the strength performance. However, the effects of unilateral SAFR on contralateral nonintervened muscle's rate of force development (RFD) are unknown. Therefore, the purpose of this investigation was to examine the acute effects of unilateral hamstrings SAFR on the contralateral limb flexibility, the isometric strength, and the RFD parameters.

Methods. Thirty-four subjects ( 21 women) completed two separate randomly sequenced experimental visits, during which the control (rested for 10 minutes) or ten, 30-second SAFR were performed with the dominant hamstring muscle group. Before (Pre) and after (Post) the interventions, the contralateral hip flexion passive ROM, the maximal explosive isometric strength of the contralateral knee flexors with the corresponding prime mover muscles' surface electromyographic $(E M G)$ amplitude were measured. Separate two-way (time $\times$ intervention) repeated measures analyses of variance (ANOVAs) were used to examine the potential changes of the dependent variables.

Results. The SAFR significantly improved the contralateral limb ROM (Pre vs. Post: $68.3 \pm 21.0$ vs. $73.2 \pm 23.2$ degrees, $p<0.001 ; d=0.22$ ). No change was found for the contralateral isometric strength or the maximal EMG amplitude. For the RFD parameters, the percent changes of the RFDs for the first 50,100, and $200 \mathrm{~ms}$ of the maximal explosive isometric contraction were $-31.2 \%,-16.8 \%$, and $-10.1 \%$, respectively, following the unilateral SAFR, relative to the control condition. In addition, the decrement of the first 50-ms RFD reached statistical significance ( $p=0.007$; Cohen's $d=0.44$ ).

Conclusion: Ten sets of 30-second unilateral hamstring SAFR improved the ROM of the nonintervened contralateral limb, but decreased its ability to generate force, especially during the early phase (e.g., $50 \mathrm{~ms}$ ) of the maximal explosive contraction. 


\section{Introduction}

77

78

79

80

81

82

83

84

85

86

87

88

89

90

91

92

93

94

95

96

97

98

99

100

101

102

103

104

105

106

107

108

109

110

111

112

113

114

115

Foam rolling is a type of massage technique that has been extensively used both in athletic and rehabilitation settings, mainly due to its portability and ease of use. Over the last decade, the increasing number of research studies have focused on the examinations of performing self-administered foam rolling (SAFR) as a pre-exercise warmup and/or a postexercise recovery strategy. These research studies have examined the short-term effects (Beardsley \& Skarabot 2015; Cheatham et al. 2015; Freiwald et al. 2016; MacDonald et al. 2013) and the potential long-term musculoskeletal adaptations (Macgregor et al. 2018) following the SAFR interventions. Briefly, SAFR consists of using one's body weight to position a specific muscle group onto a dense foam roller while moving back-and-forth to simulate soft tissue mobilization. With the undulating massage-like mechanical pressure placed upon the target muscle(s), the SAFR is an effective tool to promote soft tissue extensibility (Barnes 1997; MacDonald et al. 2013), as well as to enhance recovery from high-intensity exercise (D'Amico \& Gillis 2017; Macdonald et al. 2014; Pearcey et al. 2015). More specifically, the SAFR can improve joint range of motion (ROM) (Beardsley \& Skarabot 2015; Cheatham et al. 2015; Freiwald et al. 2016; Healey et al. 2014; Junker \& Stoggl 2015; MacDonald et al. 2013; Madoni et al. 2018; Monteiro et al. 2017; Su et al. 2017) and increase pain pressure threshold (PPT) (Cheatham \& Baker 2017; Cheatham et al. 2017; Pearcey et al. 2015) without necessarily impairing subsequent athletic performance (Behara \& Jacobson 2017; Healey et al. 2014; MacDonald et al. 2013).

In addition to the aforementioned effects that SAFR directly imposes on the local intervened muscle group, potential changes in musculoskeletal functions on the contralateral homologous or non-related heterologous (nonlocal) muscle group were also observed more recently. For example, Killen et al. showed an acute improvement on the contralateral hip flexion passive ROM after a unilateral hamstring SAFR intervention (Killen et al. 2018). Similar crossover and nonlocal effects were also reported by others for the ankle dorsiflexion ROM (Garcia-Gutierrez et al. 2018; Kelly \& Beardsley 2016). However, in a study where a small area (the sole of the foot) was rolled, no statistically significant crossover effects were demonstrated which, according to the authors, may be the result of insufficient afferent (e.g., mechanoreceptors, nociceptors, and proprioceptors) feedback from the rolling inducedmechanical pressure on tissue (Grabow et al. 2017).

Recent work suggests that foam rolling can also have nonlocal effects potentially influencing muscle mechanical sensitivity. For example, high-intensity foam rolling or rolling massage may decrease the contralateral or nonlocal muscle mechanical sensitivity (Aboodarda et al. 2015; Cavanaugh et al. 2017b; Cheatham \& Baker 2017; Cheatham et al. 2017), thereby leading to an enhancement of the stretch tolerance of these muscles. Recent work from Behm's group (Aboodarda et al. 2018; Cavanaugh et al. 2017a; Young et al. 2018) showed that the intervention of foam rolling or rolling massage could even cause the reduction of excitability at the muscular, spinal, and supraspinal levels. Thus, with the decreased corticospinal (Aboodarda et al. 2018) and spinal (Young et al. 2018) excitabilities, it is interesting and important to 
116 examine if there are any central-mediated changes on the contralateral or nonlocal muscles, due

117

118

119

120

121

122

123

124

125

126

127

128

129

130

131

132

133

134

135

136

137

138

139

140

141

142

143

144

145

146

147

148

149

150

151

152

153

154

155

to the intervention of unilateral SAFR.

Cavanaugh et al. (2017) found that three, 30-s unilateral heavy rolling massage on the calf muscle impaired the force generation during the first $200 \mathrm{~ms}$ (F200) but not the peak torque for the ipsilateral muscle maximal voluntary isometric contraction (MVIC). In addition, they also found that the F200 of the contralateral non-intervened muscle did not change after massaging the contralateral muscle (Cavanaugh et al. 2017b). It is important to mention that, during the early phase (e.g., 25-75 ms) of an explosive contraction, the RFD can be influenced by neural factors such as the maximal motor unit firing rate, the motor unit high frequency discharges (doublet) at the onset of muscular action, and the reduction in motor unit recruitment thresholds linked to central mechanisms (e.g., increased spinal motoneuron excitability) (Maffiuletti et al. 2016; Rodriguez-Rosell et al. 2018). For contraction of longer duration ( $>75 \mathrm{~ms})$, the RFD is more influenced by speed-related properties of the muscle and MVC force per se (Maffiuletti et al. 2016). Thus, examining the RFD during the first $50 \mathrm{~ms}$, or the first $100 \mathrm{~ms}$ from the onset of an explosive contraction may serve as a better and more sensitive parameter than the RFD parameters during the later phase or peak force, for monitoring potential altered excitationinduced changes in the force generating ability of the nonlocal muscles (Maffiuletti et al. 2016). In addition, these RFD parameters often serve as better predictors of motor/athletic performance than the peak force in real life scenarios such as an athletic event (Maffiuletti et al. 2016).

Therefore, the main purpose of this study was to investigate whether 10 sets of 30 -s unilateral SAFR intervention could induce potential changes in the RFD parameters (the RFD of the first $50 \mathrm{~ms}$ : $\mathrm{RFD}_{0-50}$; $\mathrm{RFD}_{0-100}$; and $\mathrm{RFD}_{0-200}$ ) of the contralateral hamstring muscle groups. This study is based on a project where other parts of the data was published previously (Killen et al. 2018). With the larger sample size from the current data pool, the effects of unilateral SAFR on the contralateral non-intervened muscle flexibility, isometric strength, as well as the prime movers' surface electromyographic (EMG) amplitude were also examined. If the contralateral $\mathrm{RFD}_{0-50}$ and $\mathrm{RFD}_{0-100}$ changed after foam rolling, then it is possible that neural mechanism(s) might have played a role influencing the contralateral non-intervened limb muscles. As shown in Cavanaugh et al. (Cavanaugh et al. 2017b), the RFD (200 $_{200}$ and the peak force of the contralateral muscle group would not likely to be statistically different following the unilateral SAFR. This work will build upon previous work by explicating the effects of high volume, long duration SAFR on musculoskeletal functions. The potential practical applications of this research may not be suitable for athletic performance, but may be of great importance for clinical practice (Cheatham et al. 2015). For instance, as physical therapists may have their patients utilize relatively high-volume, long duration SAFR to improve unilateral limb ROM, it is also important to understand the potential rolling-induced effects on the non-intervened contralateral limb muscles.

\section{Materials \& Methods}

\section{Subjects}

Peer) reviewing PDF | (2018:12:33348:2:0:NEW 7 Apr 2019) 
156

157

158

159

160

161

162

163

164

165

166

167

168

169

170

171

172

173

174

175

176

177

178

179

180

181

182

183

184

185

186

187

188

189

190

191

192

193

194

195

Thirty-four adults (male: $\mathrm{n}=13$, mean $\pm \mathrm{SD}$ age $=24 \pm 4$ years; height $=174.3 \pm 9.2 \mathrm{~cm}$; body weight $=84.3 \pm 15.2 \mathrm{~kg}$; female: $\mathrm{n}=21$, age $=21 \pm 1$ years; height $=162.8 \pm 3.5 \mathrm{~cm}$; body weight $=65.5 \pm 13.4 \mathrm{~kg}$ ) participated in this investigation. All subjects were healthy and physically active (performed resistance exercises at least once per week, and aerobic exercises at least twice per week 6 months leading up to this study). In addition, all subjects were familiar with foam rolling, and they all had the experience of performing foam rolling exercises previously. Prior to any experimental testing, each subject completed an informed consent and a pre-exercise health and exercise status questionnaire, which indicated no current or recent neuromuscular or musculoskeletal disorders in the lower body. During the consenting process, the subjects were instructed to maintain their normal habits in terms of dietary intake, hydration status, and sleep during the investigation. In addition, they were refrained from performing any upper or lower body resistance exercise at least 72 hours prior to each testing session. All experimental procedures for this investigation were approved by the University Institutional Review Board (Approval Code: 17-062).

\section{Design}

This investigation used a within-subjects randomized crossover design to examine the potential crossover effects of unilateral hamstring SAFR. Specifically, dependent variables included contralateral hip flexion passive ROM, knee flexion RFD parameters $\left(\mathrm{RFD}_{0-50}, \mathrm{RFD}_{0 \text { - }}\right.$ 100 , and $\mathrm{RFD}_{0-200}$ ) and isometric strength, as well as the hamstring muscles biceps femoris (BF) and semitendinosus (SEMI) EMG amplitude before (Pre) and immediately after (Post) the unilateral SAFR. After the first visit served as the familiarization to practice generating explosive isometric force and the SAFR protocol, the following experimental visits were conducted with a randomized order, during which the SAFR and control conditions were delivered. Between visits, a minimum of 24 hours of rest was provided. All measurements were taken from the subject's non-dominant (NONDOM) leg, and the interventions were performed on the subject's dominant (DOM) leg. Five subjects (three males) were left-footed, based on the leg dominance test (which foot the subject would kick a soccer ball).

\section{Procedures}

Upon arrival during each experimental visit, the subjects were instructed to lie down on a medical bed in the supine position for a two-minute rest. The pre-tests were then conducted as the following order: NONDOM hip flexion passive ROM (Pre-ROM), and NONDOM knee flexor isometric strength testing (Pre-MVIC). Following the baseline tests, either the control or SAFR intervention was performed. During the control visit, the subjects lay down on the same medical bed for ten minutes. During the SAFR visit, the subjects performed the same protocol as described in Killen et al. (Killen et al. 2018). Briefly, with a foam roller (FitPlus Premium HighDensity Foam Roller, Fit Plus LLC, Chattanooga, TN, USA) placed between the dominant hamstring muscle group and a yoga mat, the subject was asked to foam roll the hamstring muscles with a 1-s up (roll to the ischial tuberosity)/1-s down (roll to the popliteal fossa) tempo. 
196 During the SAFR, the DOM leg was maintained with an extended position, and the subject 197 crossed the NONDOM ankle on top of the DOM leg. Ten sets of 30-s SAFR with 30-s rest (lie 198 down on the yoga mat with both legs extended and relaxed) between sets were performed by the

199

200

201

202

203

204

205

206

207

208

209

210

211

212

213

214

215

216

217

218

219

220

221

222

223

224

225

226

227

228

229

230

231

232

233

234

235

subjects. Immediately following the intervention, tests (Post-ROM and Post-MVIC) were conducted using the exact same order and manner as during the Pre-measurements.

\section{Measurements}

Hip Flexion passive ROM

The straight leg raise test was used to measure the NONDOM hip flexion passive ROM in the supine position. With a Baseline ${ }^{\circledR}$ Bubble ${ }^{\circledR}$ inclinometer (Fabrication Enterprises Inc., White Plains, NY) placed on the NONDOM knee cap, a member of the research staff grasped the ankle of the DOM leg and raised it slowly to the point where the subjects first felt tension from the hamstring muscle group. The value from the inclinometer was then recorded as the NONDOM hip flexion passive ROM. To ensure measurement consistency, the researcher used a Sharpie pen to mark the location where the inclinometer was placed, and for subsequent measurements, the researcher placed the inclinometer in the exact same spot to perform this measurement. In addition, extra care was taken to ensure the NONDOM knee was kept straight during this procedure. At least three trials with 15 -s rest between trials were performed to establish the NONDOM hip flexion passive ROM. If the values from any two trials differed more than 2 degrees, then extra trials would be conducted. The average of the three closest trials was then calculated and recorded as the NONDOM hip flexion passive ROM.

\section{Isometric Testing (RFD and Isometric Strength)}

Following the measurement of NONDOM hip flexion passive ROM, the subjects were instructed to lie down on the same medical bed with the prone position. With both ankles hanging off the edge of the medical bed, the subjects kept the knee joints straight and relaxed. The research staff then put a cuff around the NONDOM ankle and connected the cuff to one end of a force transducer (Model SSM-AJ-500; Interface, Scottsdale, AZ, USA), with the other end of the transducer attached to a wooden platform mounted on the floor. Before testing the NONDOM knee flexor MVC strength, the subjects were instructed to perform three isometric contractions at about $50 \%$ of the perceived maximal effort to warmup. Specifically, they were told to "squeeze as fast as possible", as they practiced during the familiarization session. The subjects then performed three, 3-s MVICs of the NONDOM knee flexors with 2 minutes of recovery between the contractions. During each MVIC, the research staffs provided a verbal countdown "three, two, one, pull" to the subject, with specific emphasis on "pull as fast as possible and hard", based on the instruction recommendation by Maffiuletti et al. (Maffiuletti et al. 2016). During all maximal contractions, the research staffs provided strong verbal encouragement.

The surface EMG signals were detected through two bipolar surface EMG electrodes (input impedance $>10^{15} \Omega$, DE 2.1 Single Differential Surface EMG Sensor, Delsys, Inc., Natick, 
$236 \mathrm{MA} ; 10 \mathrm{~mm}$ interelectrode distance) placed on the NONDOM BF as well as the SEMI muscles, 237 based on the electrode placement recommendations from the SENIAM project.(Hermens et al. 238 1999) The electrodes' locations were recorded and marked with a pen to ensure the electrodes 239 were placed on the exact same spots during both experimental visits. A reference electrode (5.08 $240 \mathrm{~cm}$ diameter Dermatrode HE-R, American Imex, Irvine, CA) was placed over the $7^{\text {th }}$ cervical 241 vertebrae during data collection. Prior to detecting any EMG signals, all skin sites were shaved 242 with a razor and cleansed with rubbing alcohol. In addition, all the surface EMG sensors were 243 firmly secured to the skin with stripes of adhesive tapes.

244

245

246

247

248

249

250

251

252

253

254

255

256

257

258

259

260

261

262

263

264

265

266

267

268

269

270

271

272

273

274

275

\section{Force and Surface EMG Signal Processing}

During each MVIC trial, both the force and EMG signals were sampled at $20 \mathrm{KHz}$ with a 16-channel Bagnoli ${ }^{\mathrm{TM}}$ desktop EMG system (Delsys, Inc., Natick, MA), and stored in a laboratory computer (Dell XPS 8900, Round Rock, TX) for further analyses. The EMG signals were preamplified (gain $=1000$ ) and went through a 4th-order Butterworth filter with the bandpass set at 20 - $450 \mathrm{~Hz}$. A custom-built LabVIEW (LabVIEW, National Instruments, Austin, Texas) program was used to analyze the RFD parameters, and the peak force along with its EMG amplitude. For each MVIC, the peak force output was determined from the highest mean 500-ms portion of the force plateau during the contraction of the 3-s MVIC. The isometric strength was determined by the highest peak force output among all three MVICs. The EMG amplitude was then calculated as the root-mean-square (rms) of the same 500-ms window corresponding to the peak force of the contraction. To determine the RFD during the MVICs, we first determined the force onset point at which the force signal exceeded the baseline by $2 \%$ of the baseline-to-peak value (Andersen et al. 2010). The RFD was then calculated as the slope of the force-time curve ( $\Delta$ force/ $\Delta$ time) derived at time intervals of 0-50 $\left(\mathrm{RFD}_{0-50}\right), 0-100\left(\mathrm{RFD}_{0}\right.$ ${ }_{100}$ ), and $0-200\left(\mathrm{RFD}_{0-200}\right) \mathrm{ms}$ relative to the onset of the contraction (Aagaard et al. 2002). The RFD values from the contraction that produced the highest peak force were selected for subsequent statistical analyses.

\section{Statistical Analyses}

Test-retest reliability was calculated across the pre-values from two experimental visits by determining the intraclass correlation coefficient (ICC; relative reliability) using Model "3,1" (Weir 2005). In addition, the standard error of the measurement (SEM) was calculated for measures of absolute reliability using the equation from Hopkins (Hopkins 2000a) and Weir (Weir 2005): SEM = squat root (1-ICC). Lastly, the coefficient of variation (CV) was calculated as a normalized measure of the SEM using the equation: $\mathrm{CV}=(\mathrm{SEM} / \mathrm{Grand}$ mean $) \times 100$ (Hopkins 2000a).

Assumptions for normality of distribution for all model (fit) residuals were checked and confirmed using the Shapiro-Wilk test. Separate two-way (time [Pre vs. Post] $\times$ intervention [Control vs. SAFR]) repeated measures ANOVAs were performed to examine potential changes of all the dependent variables before and after the different interventions. When appropriate, the 
276 follow-up tests included paired t-tests with Bonferroni corrections (if there was an interaction). 277 All statistical tests were conducted using statistical software (IBM SPSS Statistics 25.0, IBM, 278 Armonk, NY) with alpha set at 0.05. In addition, effect sizes Cohen's $d$ (Cohen 1992) were 279 calculated to assess the treatment effect (Control vs. SAFR) and time effect (Pre vs. Post) for 280 each dependent variable. The Cohen's $d$ was calculated as $\left(\mathrm{Mean}_{1}-\mathrm{Mean}_{2}\right) /$ pooled Standard 281 Deviation $\left(\mathrm{SD}_{\text {pooled }}\right)$, where $\mathrm{SD}_{\text {pooled }}=$ square root $\left[\left(\mathrm{SD}_{1}{ }^{2}+\mathrm{SD}_{2}{ }^{2}\right) / 2\right]$, with 0.2, 0.6, and 1.2 as 282 the thresholds for small, medium, and large effect sizes, respectively, based on Hopkins' 283 interpretations for the magnitude of effect size (Hopkins 2000b). Lastly, separate Tufte 284

285 slopegraphs with the Cumming estimation plots (Figure 1) were generated to display the 286 complete statistical information regarding all RFD parameters before and after interventions (Control vs. SAFR) (Ho et al. 2018).

287

288

289

290

291

292

293

294

295

296

297

298

299

300

301

302

303

304

305

306

307

308

309

310

311

312

313

\section{Results}

\section{Test-retest Reliability}

Table 1 shows the ICC, SEM, and CV of measurement variables (contralateral hip flexion passive $\mathrm{ROM}$, contralateral knee flexion isometric strength, $\mathrm{RFD}_{0-50}, \mathrm{RFD}_{0-100}, \mathrm{RFD}_{0-200}$, and contralateral BF and SEMI muscle maximal EMG amplitude).

\section{NONDOM Hip Flexion Passive ROM}

The results from the two-way repeated measures ANOVA indicated that there was a statistically significant time $\times$ intervention interaction $(F(1,33)=27.371, p<0.001)$. The follow-up paired samples $t$-tests indicated that the NONDOM hip flexion passive ROM significantly increased following the SAFR (mean \pm SD: Pre vs. Post $=68.3 \pm 21.0$ vs. $73.2 \pm$ 23.2, $t=6.625, p<0.001 ; d=0.22$ ), but not following the control (Pre vs. Post $=68.4 \pm 20.7$ vs. $68.2 \pm 21.2, t=0.301, p=0.383 ; d=0.01)$. In addition, the Post-ROM value were significantly higher following the SAFR than that following the control (SAFR vs. Control $=73.2 \pm 23.2$ vs. $68.2 \pm 21.2, t=2.943, p=0.003 ; d=0.23$ ).

\section{NONDOM Knee flexors Isometric Strength and EMG Amplitude}

The two-way ANOVA showed neither an interaction $(F(1,32)=3.784, p=0.061)$ nor main effects (time and intervention) for the isometric strength of the NONDOM knee flexors. In addition, no interactions (BF: $F(1,30)=0.038, p=0.847$; SEMI: $F(1,30)=0.162, p=0.690$ ) as well as main effects (time and intervention) were found for the EMG amplitude of both BF and SEMI muscles.

\section{RFD Parameters}

For the $\mathrm{RFD}_{0-50}$, the two-way ANOVA indicated that there was a time $\times$ intervention interaction $(F(1,31)=5.834, p=0.022)$. The follow-up paired samples $t$-tests indicated that the $\mathrm{RFD}_{0-50}$ value significantly decreased following the SAFR (Pre vs. Post $=1626 \pm 1325$ vs. 1125 $315 \pm 937, t=2.630, p=0.007 ; d=0.44$ ), but not following the control (Pre vs. Post $=1756 \pm 1611$ 316 vs. $1778 \pm 1578, t=0.115, p=0.455 ; d=0.01)$. In addition, the $\mathrm{RFD}_{0-50}$ was significantly lower 
317 following the SAFR than that following the control (SAFR vs. Control $=1125 \pm 937$ vs. $1778 \pm$ $3181578, t=3.100, p=0.002 ; d=0.50)$.

319 For both $\mathrm{RFD}_{0-100}$ and $\mathrm{RFD}_{0-200}$, the results from the two-way ANOVAs did not show any 320 time $\times$ intervention interactions $\left(\mathrm{RFD}_{0-100}: F(1,31)=3.152, p=0.086 ; \mathrm{RFD}_{0-200}: F(1,31)=\right.$ $3210.872, p=0.358$ ). Figure 1 displays the individual absolute change scores (delta) of the Control 322 vs. SAFR interventions and the paired mean changes in all RFD parameters.

323

324

325

326

327

328

329

330

331

332

333

334

335

336

337

338

339

340

341

342

343

344

345

346

347

348

349

350

351

352

353

354

355

356
Insert Table 1 and Figure 1 here

\section{Discussion}

The main purpose of this investigation was to examine whether unilateral SAFR exercise could influence the contralateral muscle RFD parameters. Additionally, the contralateral limb passive ROM, and the isometric strength along with the maximal EMG amplitude were also examined. Ten, 30-s unilateral hamstring SAFR increased the contralateral passive hip flexion ROM by almost 5 degrees. In addition, the contralateral isometric strength along with the prime movers' maximal EMG amplitudes were not statistically significantly altered after the SAFR intervention. These results were consistent with the ones from our previous report (Killen et al. 2018). Regarding the RFD parameters, to our knowledge, this is the first study to investigate potential crossover effect of unilateral SAFR on the contralateral muscle RFD. Specifically, the contralateral $\mathrm{RFD}_{0-50}$ significantly decreased following the unilateral SAFR. Relative to the control condition, the SAFR imposed a small treatment effect $(d=0.42)$ on the decrement of the $\mathrm{RFD}_{0-50}$. In addition, the estimation plots with each paired mean difference (between deltaControl and deltaSAFR) suggest that the mean differences for deltaControl and deltaSAFR were below zero (mean deltaControl was less than mean deltaSAFR) for both $\mathrm{RFD}_{0-100}$ and $\mathrm{RFD}_{0-200}$ (Figure 1).

With the $\mathrm{RFD}_{0-50}$ demonstrating the statistically significant reduction, and the relatively larger mean differences for both the $\mathrm{RFD}_{0-100}$ and $\mathrm{RFD}_{0-200}$ before and after the unilateral SAFR, as compared to the control, it is also important to notice the magnitudes of the changes in these RFD parameters. Relative to the control condition, the average percent changes of the contralateral muscle $\mathrm{RFD}_{0-50}, \mathrm{RFD}_{0-100}$, and $\mathrm{RFD}_{0-200}$ following the SAFR were $-31.2 \%,-16.8 \%$, and $-10.1 \%$, respectively, showing a diminishing decline as the time interval for RFD lengthens. Folland and colleagues (Folland et al. 2014) used surface EMG to examine the relative contribution from the neural and contractile components in the early and late phases of the knee extension force-time curve. Based on their multiple linear regression analysis, the agonist muscle EMG amplitude was particularly important explaining the variance in explosive force during the initial phase (e.g., 25-75 ms). Furthermore, neural factors such as the motor unit recruitment and discharge rate, the presence of motor unit doublet discharges, the spinal motoneuron excitability, and the corticospinal excitability are also important contributing to the RFD during the initial phase of a rapid contraction (Maffiuletti et al. 2016; Rodriguez-Rosell et al. 2018). While for longer duration (e.g., > $75 \mathrm{~ms}$ ), the speed-related properties of the muscle and MVC force per se 
357 become more important for the RFD (Maffiuletti et al. 2016). Thus, the statistically significant

358

359

360

361

362

363

364

365

366

367

368

369

370

371

372

373

374

375

376

377

378

379

380

381

382

383

384

385

386

387

388

389

390

391

392

393

394

395

396

reduction for the contralateral muscle $\mathrm{RFD}_{0-50}$ might have been originated from the change(s) of one or some of the above-mentioned neural factors, induced by the unilateral SAFR.

The next obvious question is how ten, 30-s unilateral SAFR could influence the central nervous system, causing a change on the contralateral muscle RFD. In addition, another important question is where in the entire corticospinal pathway that this crossover could occur. As mentioned, repetitive high-intensity foam rolling or rolling massage exert mechanical pressure on skin, muscle, and fascia, primarily influencing mechanoreceptors and nociceptors (Behm et al. 2013), such as the type Ib Golgi tendon organs, the cutaneous receptors, and the type III/IV interstitial free nerve endings. A potential mechanism could be originated from the activation of the most abundant intrafascial mechanoreceptors, type III and IV receptors, due to the high-volume long duration of foam rolling. Specifically, nociceptive stimuli (e.g., rollinginduced mechanical pressure on soft tissue) could induce neurophysiological responses, including the influence on the descending pain modulation circuit (Vigotsky \& Bruhns 2015). In addition, diffuse noxious inhibitory control (a painful stimulus can be inhibited by another nonlocal noxious stimulus) may also serve as a central pain-modulatory mechanism influencing a nonlocal site. In the current investigation, unfortunately, it is not possible to identify the specific location that the potential crossover occurred at the corticospinal pathway, due to the lack of spinal and corticospinal excitability measurements.

Previously, Cavanaugh et al. (Cavanaugh et al. 2017b) performed three, 30-s unilateral heavy ( 7 out of 10 based on the visual analog pain scale: uncomfortable or induced some pain with most subjects) rolling massage on the subjects' calf muscle, but only found statistically significant decrease in the $\mathrm{RFD}_{0-200}$ for the ipsilateral, but not for the contralateral homologous muscle. An obvious difference between the current study and Cavanaugh et al. is the different interventions (foam rolling vs. rolling massage). However, review articles (Cheatham et al. 2015; DeBruyne et al. 2017) comparing foam rolling vs. rolling massage did not identify any intervention-related differences per se. Instead, the duration difference between these interventions might have played a more important role. Specifically, our subjects performed a total of 300 seconds foam rolls within a 10-minute period. This volume was significantly higher than the one (a total of 90 seconds 2 -s up/2-s down rolling massage) from Cavanaugh et al. (Cavanaugh et al. 2017b). Previously, Monteiro et al. (2017) compared the effects of 60 vs. 120 seconds of SAFR on joint ROM, and found longer duration/higher volume produce larger treatment effect (Monteiro et al. 2017). In addition, when implementing different SAFR durations into fatiguing resistance exercise sets (e.g. three sets of 10-repetition maximum load to failure), volumes with greater than 90 seconds were detrimental to fatigue resistance (Monteiro $\&$ Neto 2016). Thus, comparing to Cavanaugh et al. (2017b), it is possible that greater amount of the nociceptive stimulus from the current high-volume, high-intensity SAFR intervention might have induced greater inhibition, thereby showing more prominent crossover effects on the RFD parameters. In addition, the current SAFR intervention also seemed to decrease the contralateral knee flexors muscle pain perception through central pain-modulatory system, thereby leading to

Peer) reviewing PDF | (2018:12:33348:2:0:NEW 7 Apr 2019) 
397

398

399

400

401

402

403

404

405

406

407

408

409

410

411

412

413

414

415

416

417

418

419

420

421

422

423

424

425

426

427

428

429

430

431

432

433

434

435 an enhancement of the stretch tolerance of these muscles (evidenced by the increased contralateral limb ROM).

With the novel finding of the contralateral muscle RFD parameters, we do want to point out several limitations of this investigation and emphasize a caution when interpreting the results. First, we did not specifically examine the RFD parameters of the unilateral intervened muscle group, mainly due to the primary focus of this investigation was the contralateral crossover effect. Since the crossover effects were present in the non-exercised contralateral muscle RFD parameters, the unilateral rolled muscle is more likely affected. Obviously, future studies should identify the time window of the crossover effect as well as the magnitudes of changes in RFD parameters in the unilateral intervened muscle. Besides the above-mentioned limitation, our methodology also included some limitations need to be pointed out. For example, the subjects were recruited based on convenience sampling. The investigators in this study were not blinded to the measurements of the dependent variables, which could have affected the outcome measures. In addition, the SAFR intervention should have been randomly assigned between both sides of the knee flexors, for the purpose of examining potential effect of limb dominance. Lastly, caution should be taken when interpreting the results due to the high-volume of the intervention. Specifically, it may not be appropriate to apply the findings of the study to athletic field, because most SAFR sessions prior to an exercise session or a sport event do not last more than 90 seconds (e.g., 3 sets of 30-s SAFR). Thus, contralateral athletic performance such as the explosive power does not necessarily decrease following a typical SAFR with a shorter duration. Instead, our results may specifically be important for areas of physical therapy and rehabilitation, as patients may undergo longer duration of interventions.

\section{Conclusions}

A bout of ten, 30-s unilateral hamstring SAFR intervention improved the contralateral hip flexion passive ROM without altering the contralateral isometric strength performance and the prime mover muscles' maximal EMG amplitudes. However, the contralateral muscle group's ability to generate explosive force was impaired. In addition, the magnitudes of the impairments seemed to be phase-dependent (with greater impairment observed in the early phase such as the first $50 \mathrm{~ms}$ than those in the later ones such as the 100 and $200 \mathrm{~ms}$ ). These results show the evidence of contralateral crossover effects of unilateral SAFR.

\section{Acknowledgements}

The authors would like to thank all the participants who took time out of their schedules to help with this project. We also appreciate the Editor's and both reviewer' comments, which improved this manuscript significantly. 


\section{References}

437

438

439

440

441

442

443

444

445

446

447

448

449

450

451

452

453

454

455

456

457

458

459

460

461

462

463

464

465

466

467

468

469

470

471

472

473

474

475

476

477

478

479

480

481

482

483

Aagaard P, Simonsen EB, Andersen JL, Magnusson P, and Dyhre-Poulsen P. 2002. Increased rate of force development and neural drive of human skeletal muscle following resistance training. $J$ Appl Physiol (1985) 93:1318-1326. 10.1152/japplphysiol.00283.2002

Aboodarda SJ, Greene RM, Philpott DT, Jaswal RS, Millet GY, and Behm DG. 2018. The effect of rolling massage on the excitability of the corticospinal pathway. Appl Physiol Nutr Metab 43:317323. 10.1139/apnm-2017-0408

Aboodarda SJ, Spence AJ, and Button DC. 2015. Pain pressure threshold of a muscle tender spot increases following local and non-local rolling massage. BMC Musculoskelet Disord 16:265. 10.1186/s12891-015-0729-5

Andersen LL, Andersen JL, Zebis MK, and Aagaard P. 2010. Early and late rate of force development: differential adaptive responses to resistance training? Scand J Med Sci Sports 20:e162-169. 10.1111/j.1600-0838.2009.00933.x

Barnes MF. 1997. The basic science of myofascial release: morphologic change in connective tissue. $J$ Bodyw Mov Ther 1:231-238.

Beardsley C, and Skarabot J. 2015. Effects of self-myofascial release: A systematic review. J Bodyw Mov Ther 19:747-758. 10.1016/j.jbmt.2015.08.007

Behara B, and Jacobson BH. 2017. Acute Effects of Deep Tissue Foam Rolling and Dynamic Stretching on Muscular Strength, Power, and Flexibility in Division I Linemen. J Strength Cond Res 31:888892. 10.1519/JSC.0000000000001051

Behm DG, Peach A, Maddigan M, Aboodarda SJ, DiSanto MC, Button DC, and Maffiuletti NA. 2013. Massage and stretching reduce spinal reflex excitability without affecting twitch contractile properties. J Electromyogr Kinesiol 23:1215-1221. 10.1016/j.jelekin.2013.05.002

Cavanaugh MT, Aboodarda SJ, Hodgson DD, and Behm DG. 2017a. Foam Rolling of Quadriceps Decreases Biceps Femoris Activation. J Strength Cond Res 31:2238-2245. 10.1519/JSC.0000000000001625

Cavanaugh MT, Doweling A, Young JD, Quigley PJ, Hodgson DD, Whitten JH, Reid JC, Aboodarda SJ, and Behm DG. 2017b. An acute session of roller massage prolongs voluntary torque development and diminishes evoked pain. Eur J Appl Physiol 117:109-117. 10.1007/s00421-016-3503-y

Cheatham SW, and Baker R. 2017. Differences in pressure pain threshold among men and women after foam rolling. J Bodyw Mov Ther 21:978-982. 10.1016/j.jbmt.2017.06.006

Cheatham SW, Kolber MJ, Cain M, and Lee M. 2015. The Effects of Self-Myofascial Release Using a Foam Roll or Roller Massager on Joint Range of Motion, Muscle Recovery, and Performance: A Systematic Review. Int J Sports Phys Ther 10:827-838.

Cheatham SW, Stull KR, and Kolber MJ. 2017. Comparison of a vibrating foam roller and a nonvibrating foam roller intervention on knee range of motion and pressure pain threshold: A randomized controlled trial. Journal of Sport Rehabilitation:1-23.

Cohen J. 1992. A power primer. Psychol Bull 112:155-159.

D'Amico AP, and Gillis J. 2017. The influence of foam rolling on recovery from exercise-induced muscle damage. J Strength Cond Res. 10.1519/JSC.0000000000002240

DeBruyne DM, Dewhurst MM, Fischer KM, Wojtanowski MS, and Durall C. 2017. Self-Mobilization Using a Foam Roller Versus a Roller Massager: Which Is More Effective for Increasing Hamstrings Flexibility? Journal of Sport Rehabilitation 26:94-100.

Folland JP, Buckthorpe MW, and Hannah R. 2014. Human capacity for explosive force production: neural and contractile determinants. Scand J Med Sci Sports 24:894-906. 10.1111/sms.12131

Freiwald J, Baumgart C, Kühnemann M, and Hoppe MW. 2016. Foam-Rolling in sport and therapy Potential benefits and risks. Sports Orthopaedics and Traumatology 32:267-275. 10.1016/j.orthtr.2016.07.002

Peer) reviewing PDF | (2018:12:33348:2:0:NEW 7 Apr 2019) 
484

485

486

487

488

489

490

491

492

493

494

495

496

497

498

499

500

501

502

503

504

505

506

507

508

509

510

511

512

513

514

515

516

517

518

519

520

521

522

523

524

525

526

527

528

529

530

531

532
Garcia-Gutierrez MT, Guillen-Rogel P, Cochrane DJ, and Marin PJ. 2018. Cross transfer acute effects of foam rolling with vibration on ankle dorsiflexion range of motion. J Musculoskelet Neuronal Interact 18:262-267.

Grabow L, Young JD, Byrne JM, Granacher U, and Behm DG. 2017. Unilateral Rolling of the Foot did not Affect Non-Local Range of Motion or Balance. J Sports Sci Med 16:209-218.

Healey KC, Hatfield DL, Blanpied P, Dorfman LR, and Riebe D. 2014. The effects of myofascial release with foam rolling on performance. J Strength Cond Res 28:61-68. 10.1519/JSC.0b013e3182956569

Hermens H, Freriks B, Merletti R, Stegeman D, Blok J, Rau G, Disselhorst-Klug C, and Hägg G. 1999. SENIAM European recommendations for surface ElectroMyoGraphy: Result of the SENIAM Project. Enschede, The Netherlands: Roessingh Research and Development.

Ho J, Tumkaya T, Aryal S, Choi H, and Claridge-Chang A. 2018. Moving beyond P values: Everyday data analysis with estimation plots. bioRxiv:377978. 10.1101/377978

Hopkins WG. 2000a. Measures of reliability in sports medicine and science. Sports Med 30:1-15. 10.2165/00007256-200030010-00001

Hopkins WG. 2000b. A new view of statistics. Available at https://www.sportsci.org/resource/stats/effectmag.html.

Junker DH, and Stoggl TL. 2015. The Foam Roll as a Tool to Improve Hamstring Flexibility. J Strength Cond Res 29:3480-3485. 10.1519/JSC.0000000000001007

Kelly S, and Beardsley C. 2016. Specific and Cross-over Effects of Foam Rolling on Ankle Dorsiflexion Range of Motion. Int J Sports Phys Ther 11:544-551.

Killen BS, Zelizney KL, and Ye X. 2018. Crossover Effects of Unilateral Static Stretching and Foam Rolling on Contralateral Hamstring Flexibility and Strength. Journal of Sport Rehabilitation:127. 10.1123/jsr.2017-0356

Macdonald GZ, Button DC, Drinkwater EJ, and Behm DG. 2014. Foam rolling as a recovery tool after an intense bout of physical activity. Med Sci Sports Exerc 46:131-142. 10.1249/MSS.0b013e3182a123db

MacDonald GZ, Penney MD, Mullaley ME, Cuconato AL, Drake CD, Behm DG, and Button DC. 2013. An acute bout of self-myofascial release increases range of motion without a subsequent decrease in muscle activation or force. J Strength Cond Res 27:812-821. 10.1519/JSC.0b013e31825c2bc1

Macgregor LJ, Fairweather MM, Bennett RM, and Hunter AM. 2018. The Effect of Foam Rolling for Three Consecutive Days on Muscular Efficiency and Range of Motion. Sports Med Open 4:26. 10.1186/s40798-018-0141-4

Madoni SN, Costa PB, Coburn JW, and Galpin AJ. 2018. Effects of Foam Rolling on Range of Motion, Peak Torque, Muscle Activation, and the Hamstrings-to-Quadriceps Strength Ratios. J Strength Cond Res 32:1821-1830. 10.1519/JSC.0000000000002468

Maffiuletti NA, Aagaard P, Blazevich AJ, Folland J, Tillin N, and Duchateau J. 2016. Rate of force development: physiological and methodological considerations. Eur J Appl Physiol 116:10911116. 10.1007/s00421-016-3346-6

Monteiro ER, Cavanaugh MT, Frost DM, and Novaes JD. 2017. Is self-massage an effective joint rangeof-motion strategy? A pilot study. J Bodyw Mov Ther 21:223-226. 10.1016/j.jbmt.2016.10.003

Monteiro ER, and Neto VG. 2016. Effect of Different Foam Rolling Volumes on Knee Extension Fatigue. Int J Sports Phys Ther 11:1076-1081.

Pearcey GE, Bradbury-Squires DJ, Kawamoto JE, Drinkwater EJ, Behm DG, and Button DC. 2015. Foam rolling for delayed-onset muscle soreness and recovery of dynamic performance measures. $J$ Athl Train 50:5-13. 10.4085/1062-6050-50.1.01

Rodriguez-Rosell D, Pareja-Blanco F, Aagaard P, and Gonzalez-Badillo JJ. 2018. Physiological and methodological aspects of rate of force development assessment in human skeletal muscle. Clin Physiol Funct Imaging 38:743-762. 10.1111/cpf.12495

Peer) reviewing PDF | (2018:12:33348:2:0:NEW 7 Apr 2019) 
533 Su H, Chang NJ, Wu WL, Guo LY, and Chu IH. 2017. Acute Effects of Foam Rolling, Static Stretching, and Dynamic Stretching During Warm-ups on Muscular Flexibility and Strength in Young Adults. Journal of Sport Rehabilitation 26:469-477. 10.1123/jsr.2016-0102 Analgesic Implications: A Narrative Review. Pain research and treatment 2015:292805-292805. $10.1155 / 2015 / 292805$

Weir JP. 2005. Quantifying test-retest reliability using the intraclass correlation coefficient and the SEM. $J$ Strength Cond Res 19:231-240. 10.1519/15184.1

Young JD, Spence AJ, and Behm DG. 2018. Roller massage decreases spinal excitability to the soleus. $J$ Appl Physiol (1985) 124:950-959. 10.1152/japplphysiol.00732.2017 


\section{Figure 1}

Individual responses and the paired mean differences for comparisons (the absolute change scores of the Control vs. SAFR) of all three RFD parameters ( $\operatorname{RFD}_{0-50}, \mathrm{RFD}_{0-100}$, $\left.\operatorname{RFD}_{0-200}\right)$

The paired mean differences for comparisons (the absolute change scores of the Control vs. SAFR) of all three RFD parameters $\left(\mathrm{RFD}_{0-50}, \mathrm{RFD}_{0-100}, \mathrm{RFD}_{0-200}\right.$ ) are shown in the above Cumming estimation plot. The raw data (the change score) is plotted on the upper axes; each paired set of observations is connected by a line. On the lower axes, each paired mean difference is plotted as a bootstrap sampling distribution. Mean differences are depicted as dots; $95 \%$ confidence intervals $(\mathrm{Cls})$ are indicated by the ends of the vertical error bars. For each paired comparison, the paired mean difference $(\Delta)$ with $95 \% \mathrm{Cl}$ are provided as the following format: $\Delta$ [95 $\mathrm{Cl}$ : lower bound; $95 \mathrm{Cl}$ upper bound]

DeltaControl $\left(\mathrm{RFD}_{0-50}\right)$ vs. DeltaSAFR $\left(\mathrm{RFD}_{0-50}\right):-503.0$ [95 Cl: $\left.-936.0 ;-149.0\right]$; SEE $=293.89$

DeltaControl $\left(\right.$ RFD $\left._{0-100}\right)$ vs. DeltaSAFR $\left(\right.$ RFD $\left._{0-100}\right)$ : -182.0 [95 Cl: $\left.-367.0 ;-14.5\right]$; SEE $=125.94$

DeltaControl $\left(\mathrm{RFD}_{0-200}\right)$ vs. DeltaSAFR $\left(\mathrm{RFD}_{0-200}\right):-64.8$ [95 Cl: $\left.-225.0 ; 29.6\right] ;$ SEE $=67.99$

Note: RFD = rate of force development; SAFR = self-administered foam rolling; SEE = standard error of the estimate 

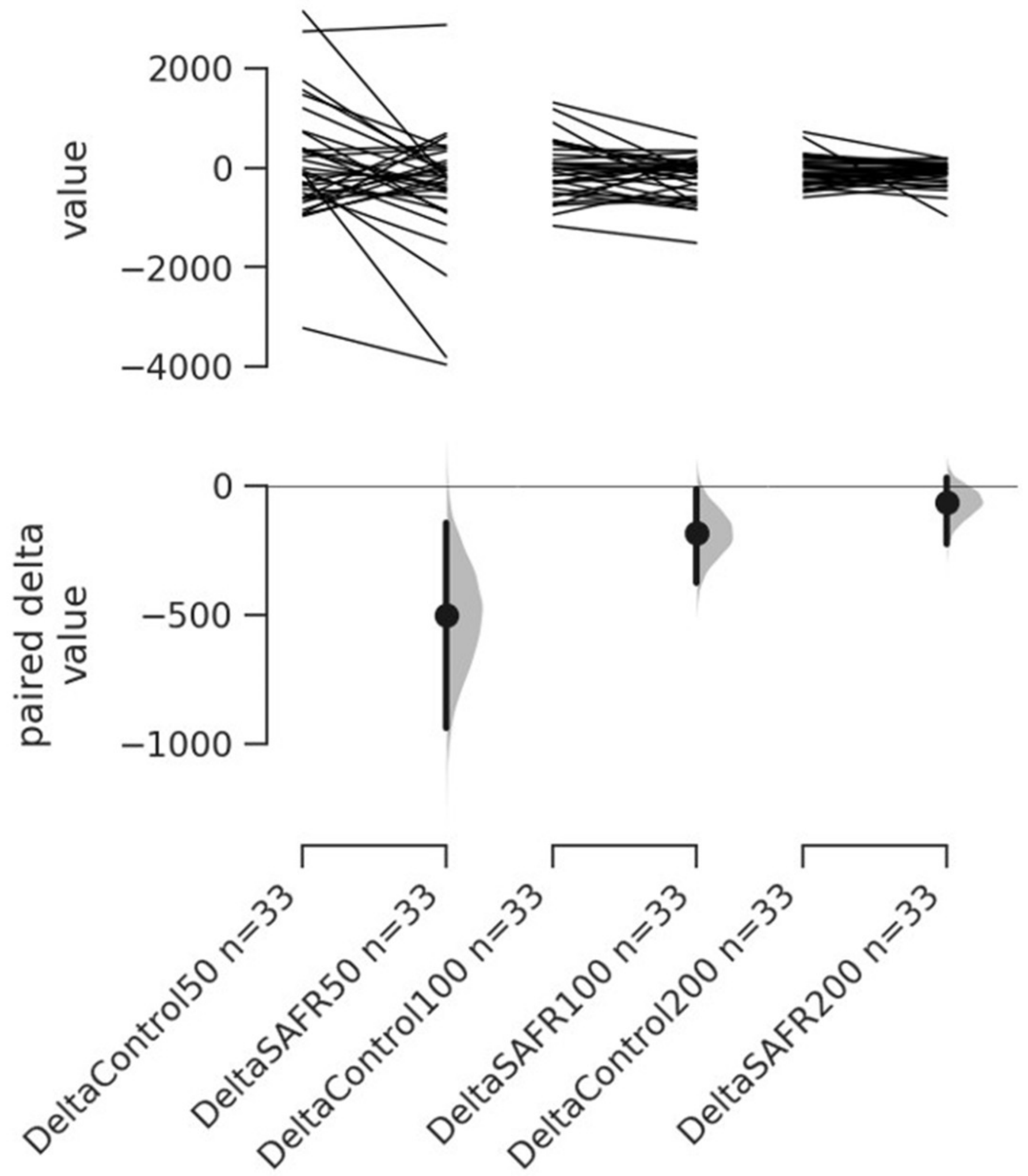


\section{Table 1 (on next page)}

Test-retest reliability for dependent variables

The intraclass correlation coefficient (model " 3,1 ") (ICC $(3,1)$ ), standard error of measurement (SEM), and coefficient of variation (CV) of variable measurements (the contralateral hip flexion passive range of motion [ROM], the contralateral biceps femoris [BF] and semitendinosus [SEMI] EMG amplitude, the contralateral knee flexion isometric strength, and the rate of force development (RFD) for the first 50 [RFD ${ }_{0-50}$ ], 100 [RFD $0-100$ ], and 200 [RFD $0-200$ ] ms of the maximal isometric contraction). 
1 TABLE 1. The intraclass correlation coefficient (model " 3,1 ") (ICC $(3,1)$ ), standard error of

2 measurement (SEM), and coefficient of variation (CV) of variable measurements (the

3 contralateral hip flexion passive range of motion [ROM], the contralateral biceps femoris [BF]

4 and semitendinosus [SEMI] EMG amplitude, the contralateral knee flexion isometric strength,

5 and the rate of force development (RFD) for the first $50\left[\mathrm{RFD}_{0-50}\right], 100\left[\mathrm{RFD}_{0-100}\right]$, and 200

$6\left[\mathrm{RFD}_{0-200}\right] \mathrm{ms}$ of the maximal isometric contraction).

\begin{tabular}{l|l|l|l|}
\hline Variable Measures & ICC $(3,1)$ & SEM & CV (SEM \%) \\
\hline Passive ROM $\left(^{\circ}\right)$ & 0.91 & 4.26 & $6.2 \%$ \\
\hline Isometric Strength $(\mathrm{N})$ & 0.88 & 34.27 & $10.6 \%$ \\
\hline EMG Amplitude of BF $(\mu \mathrm{V})$ & 0.84 & 31.76 & $23.8 \%$ \\
\hline EMG Amplitude of SEMI $(\mu \mathrm{V})$ & 0.80 & 38.55 & $30.2 \%$ \\
\hline $\mathrm{RFD}_{0-50}(\mathrm{~N} / \mathrm{s})$ & 0.81 & 633.00 & $37.9 \%$ \\
\hline $\mathrm{RFD}_{0-100}(\mathrm{~N} / \mathrm{s})$ & 0.84 & 312.39 & $23.5 \%$ \\
\hline $\mathrm{RFD}_{0-200}(\mathrm{~N} / \mathrm{s})$ & 0.87 & 184.06 & $17.2 \%$ \\
\hline
\end{tabular}

7

8

9 